\title{
The Integral Analog of the Leibniz Rule
}

\section{By Thomas J. Osler}

Abstract. This paper demonstrates that the classical Leibniz rule for the derivative of the product of two functions

$$
D^{N} u v=\sum_{k=0}^{N}\left(\begin{array}{l}
N \\
k
\end{array}\right) D^{N-k} u D^{k} v
$$

has the integral analog

$$
D^{\alpha} u v=\int_{-\infty}^{\infty}\left(\begin{array}{l}
\alpha \\
\omega
\end{array}\right) D^{\alpha-\omega} u D^{\omega} v d \omega .
$$

The derivatives occurring are "fractional derivatives." Various generalizations of the integral are given, and their relationship to Parseval's formula from the theory of Fourier integrals is revealed. Finally, several definite integrals are evaluated using our results.

1. Introduction. The derivative of the function $f(z)$ with respect to $g(z)$ of arbitrary (real or complex) order $\alpha$ is denoted by $D_{\sigma(z)}^{\alpha} f(z)$, and called a "fractional derivative". It is a generalization of the familiar derivative $d^{\alpha} f(z) /(d g(z))^{\alpha}$ to values of $\alpha$ which are not natural numbers. In previous papers (see [3] [9]), the author discussed extensions to fractional derivatives of certain rules and formulas for ordinary derivatives familiar from the elementary calculus. This paper continues the previous study and presents integral analogs of the familiar Leibniz rule for the derivative of a product

$$
D^{N} u(z) v(z)=\sum_{k=0}^{N}\left(\begin{array}{l}
N \\
k
\end{array}\right) D^{N-k} u(z) D^{k} v(z) .
$$

We present below continually more complex extensions of the Leibniz rule.

Case 1. The simplest integral analog of the Leibniz rule is

$$
D_{z}^{\alpha} u(z) v(z)=\int_{-\infty}^{\infty}\left(\begin{array}{l}
\alpha \\
\omega
\end{array}\right) D_{z}^{\alpha-\omega} u(z) D_{z}^{\omega} v(z) d \omega,
$$

where $\left(\begin{array}{c}\alpha \\ \omega\end{array}\right)=\Gamma(\alpha+1) / \Gamma(\alpha-\omega+1) \Gamma(\omega+1)$, and $\alpha$ is any real or complex number. Notice that we integrate over the order of the derivatives.

Case 2. (1.1) can be generalized to the case where we differentiate with respect to an arbitrary function $g(z)$, and $\omega$ is replaced by $\omega+\gamma$, where $\gamma$ is arbitrary (real or complex).

Received March 23, 1972.

AMS 1970 subject classifications. Primary 26A33, 44A45; Secondary 33A30, 42A68.

Key words and phrases. Fractional derivative, Leibniz rule, Fourier transforms, Parseval relation, special functions.

Copyright @ 1972, American Mathematical Society 


$$
D_{o(z)}^{\alpha} u(z) v(z)=\int_{-\infty}^{\infty}\left(\begin{array}{c}
\alpha \\
\omega+\gamma
\end{array}\right) D_{o(z)}^{\alpha-\omega-\gamma} u(z) D_{o(z)}^{\omega+\gamma} v(z) d \omega .
$$

Case 3. The product $u(\xi) v(\zeta)$ can be replaced by an arbitrary function of two variables $f(\xi, \zeta)$ :

$$
D_{g(z)}^{\alpha} f(z, z)=\left.\int_{-\infty}^{\infty}\left(\begin{array}{c}
\alpha \\
\omega+\gamma
\end{array}\right) D_{g(\xi), g(\zeta)}^{\alpha-\omega-\gamma, \omega+\gamma} f(\xi, \zeta)\right|_{\xi=z ; \zeta=z} d \omega
$$

where $D_{z, w}^{a, b}$ is a generalization of the "partial derivative" operator $\partial^{a+b} /(\partial z)^{a}(\partial w)^{b}$.

Case 4. Our most general integral form of the Leibniz rule is

$$
D_{o(z)}^{\alpha} f(z, z)=\int_{-\infty}^{\infty} I(\omega) d \omega
$$

where

$$
I(\omega)=\left.\left(\begin{array}{c}
\alpha \\
\omega+\gamma
\end{array}\right) D_{g(\xi), \sigma(\zeta)}^{\alpha-\omega-\gamma, \omega+\gamma}\left[f(\xi, \zeta) \theta_{g(\zeta)}(\zeta ; z) q(\xi)^{\omega+\gamma} q(\zeta)^{-\omega-\gamma-1}\right]\right|_{\xi=z ; \zeta=z} d \omega,
$$

and where $\theta(\zeta ; z)=(g(\zeta)-g(z)) q(\zeta)$. The three previous integrals are all special cases of (1.4). The author's series form of the Leibniz rule [7]

$$
D_{o(z)}^{\alpha} f(z, z)=\sum_{n=-\infty}^{\infty} I(a n) a
$$

suggests the validity of (1.4) by letting $a \rightarrow 0$ (in which case $a$ becomes $d \omega$, an becomes $\omega$, and $\sum$ becomes $\left.\int\right)$. If $f\left(\xi, g^{-1}(0)\right)=0,(1.4)$ assumes the simpler form

$$
D_{o(z)}^{\alpha} f(z, z)=\left.\int_{-\infty}^{\infty}\left(\begin{array}{c}
\alpha \\
\omega+\gamma
\end{array}\right) D_{o(\xi), \sigma(\zeta)}^{\alpha-\omega-\gamma, \omega+\gamma}\left[\frac{\partial f(\xi, \zeta)}{\partial g(\zeta)}\left\{\frac{q(\xi)}{q(\zeta)}\right\}^{\omega+\gamma}\right]\right|_{\xi=z ; \zeta=z} d \omega .
$$

In previous papers [5], [7], [8], the author demonstrated that formulas familiar from Fourier analysis are closely related to formulas involving fractional derivatives. In this paper, we show that the familiar Parseval's integral formula [11, p. 50] is, in a certain sense, a special case of our integral analog of the Leibniz rule (1.4).

A proof of (1.4) is presented, and the region in the $z$-plane in which the integral converges is revealed.

Finally, several examples of (1.4) are given for specific functions $f(\xi, \zeta), g(z)$ and $q(\zeta)$. A table of definite integrals (Table 5.2) emerges.

2. Fractional Derivatives. In a previous paper [3], the author explored the definitions of the fractional derivatives of a function in detail. In this section, we merely review the definitions so as to make the paper self-contained. We assume that the reader is familiar with the motivation provided in [3].

The most common definition for the fractional derivative of order $\alpha$ found in the literature is the "Riemann-Liouville integral"

$$
D_{z}^{\alpha} f(z)=\Gamma(-\alpha)^{-1} \int_{0}^{z} f(t)(z-t)^{-\alpha-1} d t,
$$

where $\operatorname{Re}(\alpha)<0$. The concept of a fractional derivative with respect to an arbitrary function $g(z), D_{g(z)}^{\alpha} f(z)$, was apparently introduced for the first time in the author's paper [3], while the idea appeared earlier for certain specific functions. The most 
convenient form of the definition for our purposes is given through a generalization of Cauchy's integral formula.

Definition 2.1. Let $f(z)$ be analytic in the simply connected region $R$. Let $g(z)$ be regular and univalent on $R$, and let $g^{-1}(0)$ be an interior or boundary point of $R$. Assume also that $\int_{C} f(z) g^{\prime}(z) d z=0$ for any simple closed contour $C$ in $R \cup\left\{g^{-1}(0)\right\}$ through $g^{-1}(0)$. Then if $\alpha$ is not a negative integer, and $z$ is in $R$, we define the fractional derivative of order $\alpha$ of the function $f(z)$ with respect to $g(z)$ to be

$$
D_{g(z)}^{\alpha} f(z)=\frac{\Gamma(\alpha+1)}{2 \pi i} \int_{0^{-1}(0)}^{\left(z^{+}\right)} \frac{f(\zeta) g^{\prime}(\zeta) d \zeta}{(g(\zeta)-g(z))^{\alpha+1}} .
$$

For nonintegral $\alpha$, the integrand has a branch line which begins at $\zeta=z$ and passes through $\zeta=g^{-1}(0)$. The limits of integration imply that the contour of integration starts at $g^{-1}(0)$, encloses $z$ once in the positive sense, and returns to $g^{-1}(0)$ without cutting the branch line or leaving $R \cup\left\{g^{-1}(0)\right\}$.

If $\alpha$ is a negative integer $-N, \Gamma(\alpha+1)=\infty$ while the integral in (2.1) vanishes. If we interpret $(2.1)$ as the limit as $\alpha$ approaches $-N$, it then defines the derivative of order $-N$, or perhaps we should say the " $N$ th iterated integral of $f(z)$ with respect to $g(z)$ "'.

It is important to notice that with the restrictions on $g(z)$ as given in Definition 2.1 , the substitution $w=g(z)$ maintains the equality $D_{w}^{\alpha} f(w)=D_{o(z)}^{\alpha} f(g(z))$.

It is particularly interesting to set $g(z)=z-a$, for we find that

$$
D_{z-a}^{\alpha} f(z)=\frac{\Gamma(\alpha+1)}{2 \pi i} \int_{a}^{(z+)} f(\zeta)(\zeta-z)^{-\alpha-1} d \zeta .
$$

While ordinary derivatives with respect to $z$ and $z-a$ are equal, (2.2) shows that this is not the case for fractional derivatives, since the value of the contour integral depends on the point $\zeta=a$ at which the contour crosses the branch line.

We also require fractional partial derivatives.

Definition 2.2. Let $f(z, w)$ be an analytic function of two variables for $z$ and $w$ in the simply connected region $R$. Let $g(z)$ be regular and univalent on $R$, and let $g^{-1}(0)$ be an interior or boundary point of $R$. Assume also that $\int_{C} f(z, w) g^{\prime}(w) d w=0$ and $\int_{C} D_{o(w)}^{\beta} f(z, w) g^{\prime}(z) d z=0$ for any simple closed contour $C$ in $R \cup\left\{g^{-1}(0)\right\}$ through $g^{-1}(0)$. Then if $\alpha$ and $\beta$ are not negative integers, and $z$ and $w$ are in $R$ we write

$$
\begin{aligned}
& D_{o(z), o(w)}^{\alpha, \beta} f(z, w)=D_{o(z)}^{\alpha}\left[D_{o(w)}^{\beta} f(z, w)\right] \\
& \quad=\frac{\Gamma(\alpha+1) \Gamma(\beta+1)}{-4 \pi^{2}} \int_{\sigma^{-1}(0)}^{(z+)} \frac{g^{\prime}(\zeta)}{(g(\zeta)-g(z))^{\alpha+1}} \int_{o^{-1}(0)}^{\left(w^{+}\right)} \frac{f(\zeta, \xi) g^{\prime}(\xi) d \xi d \zeta}{(g(\xi)-g(w))^{\beta+1}} .
\end{aligned}
$$

Note. The expression $(g(\zeta)-g(z))^{\alpha+1}=\exp [(\alpha+1) \ln (g(\zeta)-g(z))]$ appearing in the above definitions is in general a multiple-valued function. To remove this uncertainty, select a branch cut which starts at $\zeta=z$ and passes through $\zeta=g^{-1}(0)$ in the $\zeta$-plane. Then let $\ln (g(\zeta)-g(z))$ assume values which are continuous and single-valued on the cut $\zeta$-plane and let $\ln (g(\zeta)-g(z))$ be real when $g(\zeta)-g(z)$ is positive.

3. Connection with Fourier Analysis. In this section, we show that the special case of our integral analog of the Leibniz rule (1.1) is formally a generalization of 
the Parseval's formula [11, p. 50] familiar from the study of Fourier integrals.

Consider the definition of fractional derivatives (2.2), with $a=0$. Take the contour of integration to be the circle parametrized by the variable " $t$ ":

$$
\zeta=z+z e^{i t}, \text { where }-\pi<t<\pi .
$$

We obtain at once

$$
\frac{z^{\alpha} D_{z}^{\alpha} f(z)}{\Gamma(\alpha+1)}=\frac{1}{2 \pi} \int_{-\pi}^{\pi} f\left(z+z e^{i t}\right) e^{-i t \alpha} d t
$$

If we set

$$
\begin{aligned}
f[t] & =f\left(z+z e^{i t}\right) & & \text { for }-\pi<t<\pi, \\
& =0 & & \text { otherwise, }
\end{aligned}
$$

and denote the Fourier transform of $f[t]$ by

$$
f^{*}(\alpha)=\frac{1}{2 \pi} \int_{-\infty}^{\infty} f[t] e^{-i t \alpha} d t
$$

we see that (3.2) becomes

$$
z^{\alpha} D_{z}^{\alpha} f(z) / \Gamma(\alpha+1)=f^{*}(\alpha)
$$

Thus, we see that the fractional derivative $D_{z}^{\alpha} f(z)$ (modulo a multiplicative factor) is in a certain sense a generalization of the Fourier transform. By varying $z$, the circle (3.1) changes and, thus, the function $f[t]$ changes. We say that we have "extended the Fourier integral into the complex $z$-plane".

Now, consider (1.1) written in the form

$$
\frac{z^{\alpha} D_{z}^{\alpha} u(z) v(z)}{\Gamma(\alpha+1)}=\int_{-\infty}^{\infty} \frac{z^{\alpha-\omega} D_{z}^{\alpha-\omega} u(z)}{\Gamma(\alpha-\omega+1)} \frac{z^{\omega} D_{z}^{\omega} v(z)}{\Gamma(\omega+1)} d \omega .
$$

Using (3.2), we get

$$
\begin{aligned}
\frac{1}{2 \pi} \int_{-\pi}^{\pi} & u\left(z+z e^{i t}\right) e^{-i t \alpha} v\left(z+z e^{i t}\right) d t \\
& =\int_{-\infty}^{\infty}\left\{\frac{1}{2 \pi} \int_{-\pi}^{\pi} u\left(z+z e^{i t}\right) e^{-i t \alpha} e^{i \omega t} d t \frac{1}{2 \pi} \int_{-\pi}^{\pi} v\left(z+z e^{i t}\right) e^{-i \omega t} d t\right\} d \omega .
\end{aligned}
$$

Using the notation

$$
\begin{aligned}
u[t] & =u\left(z+z e^{i t}\right) e^{-i t \alpha} & & \text { for }-\pi<t<\pi, \\
& =0 & & \text { otherwise, } \\
v[t] & =v\left(z+z e^{i t}\right) & & \text { for }-\pi<t<\pi, \\
& =0 & & \text { otherwise, }
\end{aligned}
$$

and the notation (3.3) for Fourier transforms, we convert (3.5) into

$$
\frac{1}{2 \pi} \int_{-\infty}^{\infty} u[t] v[t] d t=\int_{-\infty}^{\infty} u^{*}(-\omega) v^{*}(\omega) d \omega
$$


(3.6) is the Parseval's formula [11, p. 50]. Thus, our integral analog of the Leibniz rule "extends the Parseval's formula into the complex $z$-plane".

We conclude this section by reviewing three connections observed in previous papers between formulas from Fourier analysis, and new formulas involving fractional derivatives.

1. The familiar Fourier series

$$
f[t]=\sum_{n=-\infty}^{\infty} f_{n} e^{i a n t}
$$

is (in a sense similar to that above) a special case of the generalized Taylor's series [5]

$$
f(z)=\sum_{n=-\infty}^{\infty} \frac{\left.a D_{z-b}^{a n+\gamma} f(z)\right|_{z=z_{0}}}{\Gamma(a n+\gamma+1)}\left(z-z_{0}\right)^{a n+\gamma} .
$$

2. The series form of the Parseval's relation

$$
\frac{a}{2 \pi} \int_{-\pi / a}^{\pi / a} u[t] v[t] d t=\sum_{n=-\infty}^{\infty} \frac{a}{2 \pi} \int_{-\pi / a}^{\pi / a} u[t] e^{i a n t} d t \frac{a}{2 \pi} \int_{-\pi / a}^{\pi / a} v[t] e^{-i a n t} d t,
$$

is a special case of the series form of the Leibniz rule [7]

$$
D_{z}^{\alpha} u(z) v(z)=\sum_{n=-\infty}^{\infty} a\left(\begin{array}{c}
\alpha \\
a n+\gamma
\end{array}\right) D_{z}^{\alpha-a n-\gamma} u(z) D_{z}^{a n+\gamma} v(z) .
$$

3. The Fourier integral theorem

$$
f[t]=\int_{-\infty}^{\infty} \frac{1}{2 \pi} \int_{-\infty}^{\infty} f[\xi] e^{-i \xi \omega} d \xi e^{i \omega t} d \omega
$$

is a special case of the integral analog of Taylor's series [8]

$$
f(z)=\int_{-\infty}^{\infty} \frac{\left.D_{z-b}^{\omega+\gamma} f(z)\right|_{z=z_{0}}}{\Gamma(\omega+\gamma+1)}\left(z-z_{0}\right)^{\omega+\gamma} d \omega .
$$

We now leave our discussion of Fourier analysis, and present a rigorous derivation of our integral analog of the Leibniz rule.

4. Rigorous Derivations. In the previous section, we saw that our integral analog of the Leibniz rule is formally related to the integral form of Parseval's relation from the theory of Fourier transforms. It would seem natural then that a rigorous derivation would follow from known results on Fourier integrals. While this is easily achieved for functions $f(z, w)$ of a particular class, a derivation by this method sufficient to cover all $f(z, w)$ of interest has escaped the author. It appears our integral form of the Leibniz rule is more difficult to prove than the series form (1.5). In particular, it is necessary to restrict $f(z, w)$ in the following Theorem 4.1 (see (i)) to a narrower class of functions than was necessary for the series form of the Leibniz rule in which only the growth of $f$ at the origin was restricted by $|f(z, w)| \leqq$ $M|z|^{P}|z|^{Q}$, for $P, Q$, and $P+Q$ in the interval $(-1, \infty)$ [9].

THEOREM 4.1. (i) Let $f(\xi, \zeta)=\xi^{P} \zeta^{Q} F(\xi$, $\zeta)$, where $\operatorname{Re}(P), \operatorname{Re}(Q)$, and $\operatorname{Re}(P+Q)$ are in the interval $(-1, \infty)$, and let $F(\xi, \zeta)$ be analytic for $\xi$ and $\zeta$ in the simply connected open subset of the complex plane $R$, which contains the origin. 
(ii) Let $\theta(\zeta ; z)=(\zeta-z) q(\zeta)$ be a given univalent analytic function of $\zeta$ on $R$ such that $q(\zeta)$ is never zero on $R$.

(iii) Assume that the curves $C(z)=\{\zeta|| \theta(\zeta ; z)|=| \theta(0 ; z) \mid\}$ are simple and closed for each $z$ such that $C(z) \subset R$.

(iv) Call $S=\{z \mid C(z) \subset R\}$.

Then for $z \in S$, and all real or complex $\alpha$ and $\gamma$, the integral analog of the Leibniz rule is

$$
D_{z}^{\alpha} f(z, z)=\int_{-\infty}^{\infty} I(\omega) d \omega
$$

where

$$
I(\omega)=\left.\left(\begin{array}{c}
\alpha \\
\omega+\gamma
\end{array}\right) D_{\xi, \zeta}^{\alpha-\omega-\gamma, \omega+\gamma}\left[f(\xi, \zeta) \theta_{\zeta}(\zeta ; z) q(\xi)^{\omega+\gamma} q(\zeta)^{-\omega-\gamma-1}\right]\right|_{\xi=z ; \zeta=z}
$$

and $\theta_{\zeta}(\zeta ; z)=d \theta(\zeta ; z) / d \zeta$.

Remark.

$$
\left(\begin{array}{c}
\alpha \\
\omega+\gamma
\end{array}\right)=\Gamma(\alpha+1) / \Gamma(\alpha-\omega-\gamma+1) \Gamma(\omega+\gamma+1)
$$

is, in general, undefined when $\alpha$ is a negative integer. In this case, dividing both sides of $(4.1)$ by $\Gamma(\alpha+1)$ produces a valid expression.

Proof. The $C(z)$ are the curves in the complex $\zeta$-plane which pass through the origin, over which the amplitude of $\theta(\zeta ; z)$ is constant. For example, if $\theta(\zeta ; z)=$ $\zeta-z$, then $C(z)$ is the circle centered at $\zeta=z$ passing through the origin. By restricting $z$ to $S$ (described in (iv)), we insure that the curves $C(z)$ are contained in the region $R$ on which $f(\xi, \zeta)$ is sufficiently regular for the manipulations which follow.

Using Definition 2.2 of fractional differentiation, we can express $I(\omega)$ as

$$
I(\omega)=\frac{\Gamma(\alpha+1)}{(2 \pi i)^{2}} \int_{0}^{\left(z^{+}\right)} \int_{0}^{\left(z^{+}\right)} \frac{f(\xi, \zeta) \theta_{\zeta}(\zeta ; z) q(\xi)^{\omega+\gamma} q(\zeta)^{-\omega-\gamma-1} d \zeta d \xi}{(\xi-z)^{\alpha-\omega-\gamma+1}(\zeta-z)^{\omega+\gamma+1}},
$$

and since $\theta(\zeta ; z)=q(\zeta)(\zeta-z)$ and $f(\xi, \zeta)=\xi^{P} \zeta^{Q} F(\xi, \zeta)$, we get

$$
I(\omega)=\frac{\Gamma(\alpha+1)}{(2 \pi i)^{2}} \int_{0}^{(z+)} \int_{0}^{\left(z^{+}\right)} \frac{\xi^{P} \zeta^{0} F(\xi, \zeta) q(\xi)^{\alpha+1} \theta_{\xi}(\xi ; z) \theta_{\zeta}(\zeta ; z) d \zeta d \xi}{\theta(\xi ; z)^{\alpha-\omega-\gamma+1} \theta(\zeta ; z)^{\omega+\gamma+1} \theta_{\xi}(\xi ; z)}
$$

Make the substitutions

$$
t-z=\theta(\zeta ; z), \quad s-z=\theta(\xi ; z)
$$

and call $b=z+\theta(0 ; z)$. Since $\theta(\xi ; z)$ is a univalent function of $\xi$, we have

$$
\frac{\xi^{P} \zeta^{Q} F(\xi, \zeta) q(\xi)^{\alpha+1}}{\theta_{\xi}(\xi ; z)}=(s-z)^{P}(t-z)^{Q} \mathcal{F}(s, t)
$$

where $\theta_{\xi}(\xi ; z)$ is not zero and, thus, $\mathcal{F}(s, t)$ is an analytic function. The curve $C(z)$ has now become the circle $|t-z|=|\theta(0 ; z)|=|b-z|$ in the $t$-plane, and $|s-z|=$ $|b-z|$ in the $s$-plane. Thus, if $z \in S$, then $\mathcal{F}(s, t)$ is analytic for $s$ and $t$ inside and on $|t-z|=|b-z|$ and $|s-z|=|b-z|$ and so

$$
\mathcal{F}(s, t)=\sum_{m=0}^{\infty} \sum_{n=0}^{\infty} a_{m n}(s-z)^{m}(t-z)^{n}
$$


where the series converges absolutely and uniformly inside and on the circles just mentioned. Thus, (4.2) becomes

$$
I(\omega)=\frac{\Gamma(\alpha+1)}{(2 \pi i)^{2}} \int_{b}^{\left(z^{+}\right)} \int_{b}^{\left(z^{+}\right)} \frac{(s-b)^{P}(t-b)^{Q} \mathcal{F}(s, t) d t d s}{(s-z)^{\alpha-\omega-\gamma+1}(t-z)^{\omega+\gamma+1}} .
$$

Substituting the series for $\mathcal{F},(4.5)$, into this last integral, we can then integrate termwise since we have a uniformly convergent series times an integrable factor. We get

$$
\begin{aligned}
I(\omega)=\sum_{m, n=0}^{\infty} & \frac{\Gamma(\alpha+1) a_{m n}}{\Gamma(\alpha-\omega-\gamma-m+1) \Gamma(\omega+\gamma-n+1)} \\
& \frac{\Gamma(\alpha-\omega-\gamma-m+1)}{2 \pi i} \int_{b}^{(z+)} \frac{(s-b)^{P} d s}{(s-z)^{\alpha-\omega-\gamma-m+1}} \\
& \frac{\Gamma(\omega+\gamma-n+1)}{2 \pi i} \int_{b}^{(z+)} \frac{(t-b)^{Q} d t}{(t-z)^{\omega+\gamma-n+1}} .
\end{aligned}
$$

It is well known that $[6$, p. 647]

$$
\frac{\Gamma(B+1)}{2 \pi i} \int_{b}^{\left(z^{+}\right)} \frac{(s-b)^{P} d s}{(s-z)^{B+1}}=D_{z-b}^{B}(z-b)^{P}=\frac{\Gamma(P+1)(z-b)^{P-B}}{\Gamma(P-B+1)}
$$

and, thus, we get

$$
I(\omega)=\sum_{m, n=0}^{\infty} A_{m n} G(\omega+m) H(\omega-n)
$$

where

$$
\begin{aligned}
A_{m n} & =\Gamma(\alpha+1) \Gamma(P+1) \Gamma(Q+1) a_{m n}(z-b)^{P+Q-\alpha+m+n}, \\
G(\omega+m) & =\frac{1}{\Gamma(\alpha-\gamma-\omega-m+1) \Gamma(P-\alpha+\gamma+\omega+m+1)} \\
H(\omega-n) & =\frac{1}{\Gamma(\gamma+\omega-n+1) \Gamma(Q-\gamma-\omega+n+1)} .
\end{aligned}
$$

Assume for the moment that we can integrate (4.7) term by term (we will prove this later) to get

$$
\int_{-\infty}^{\infty} I(\omega) d \omega=\sum_{m, n=0}^{\infty} A_{m n} \int_{-\infty}^{\infty} G(\omega+m) H(\omega-n) d \omega .
$$

From [1, Vol. 2, p. 300, (21)], we get

$$
\begin{aligned}
\int_{-\infty}^{\infty} G(\omega+ & m) H(\omega-n) d \omega \\
& =\frac{\Gamma(P+Q+1)}{\Gamma(P+1) \Gamma(Q+1) \Gamma(\alpha-m-n+1) \Gamma(P+Q-\alpha+m+n+1)}
\end{aligned}
$$

and, thus, (4.8) becomes

$$
\int_{-\infty}^{\infty} I(\omega) d \omega=\sum_{m, n=0}^{\infty} \frac{\Gamma(\alpha+1) \Gamma(P+Q+1) a_{m n}(z-b)^{P+Q-\alpha+m+n}}{\Gamma(\alpha-m-n+1) \Gamma(P+Q-\alpha+m+n+1)} .
$$


Using (4.6), we can write (4.9) as

$$
\int_{-\infty}^{\infty} I(\omega) d \omega=\sum_{m, n=0}^{\infty} \frac{a_{m n} \Gamma(\alpha+1)}{2 \pi i} \int_{b}^{\left(z^{+}\right)} \frac{(s-b)^{P+Q} d s}{(s-z)^{\alpha-m-n+1}} .
$$

We can interchange summation and integration

$$
\int_{-\infty}^{\infty} I(\omega) d \omega=\frac{\Gamma(\alpha+1)}{2 \pi i} \int_{b}^{\left(z^{+}\right)} \frac{(s-b)^{P+Q}}{(s-z)^{\alpha+1}} \sum_{m, n=0}^{\infty} a_{m n}(s-z)^{m+n} d s,
$$

since we have an integrable factor times a uniformly convergent power series. Using (4.5), we get

$$
\int_{-\infty}^{\infty} I(\omega) d \omega=\frac{\Gamma(\alpha+1)}{2 \pi i} \int_{b}^{\left(z^{+}\right)} \frac{(s-b)^{P+Q} \mathcal{F}(s, s) d s}{(s-z)^{\alpha+1}},
$$

and using (4.3) and (4.4), we can rewrite this as

$$
\begin{aligned}
\int_{-\infty}^{\infty} I(\omega) d \omega & =\frac{\Gamma(\alpha+1)}{2 \pi i} \int_{0}^{\left(z^{+}\right)} \frac{f(\xi, \xi) q(\xi)^{\alpha+1} \theta_{\xi}(\xi ; z) d \xi}{\theta(\xi ; z)^{\alpha+1} \theta_{\xi}(\xi ; z)} \\
& =\frac{\Gamma(\alpha+1)}{2 \pi i} \int_{0}^{(z+)} \frac{f(\xi, \xi) d \xi}{(\xi-z)^{\alpha+1}}=D_{z}^{\alpha} f(z, z) .
\end{aligned}
$$

Thus, the theorem is proved as soon as we verify the term by term integration in (4.8).

We know that (4.8) is correct provided

$$
\sum_{m, n=0}^{\infty}\left|A_{m n}\right| \int_{-\infty}^{\infty}|G(\omega+m) H(\omega-n)| d \omega
$$

converges [10, p. 45]. It is well known that $\Gamma(a-\omega-1)^{-1} \Gamma(\omega+b)^{-1}$ is an entire function of $\omega$ and that [2, Vol. 1, p. 33, (11)]

$\frac{1}{\Gamma(a-\omega+1) \Gamma(\omega+b)}$

$$
\begin{aligned}
& =\frac{\Gamma(\omega-a) \sin \pi(\omega-a)}{\Gamma(\omega+b) \pi}=\omega^{-a-b} \sin \pi(\omega-a) A(\omega) / \pi \\
& \text { where } A(\omega) \rightarrow 1 \text { as } \omega \rightarrow+\infty ; \text { and } \\
& =\frac{\Gamma(1-\omega-b) \sin \pi(b+\omega)}{\Gamma(a-\omega+1) \pi}=(-\omega)^{-a-b} \sin \pi(\omega+b) B(\omega) / \pi \\
& \text { where } B(\omega) \rightarrow 1 \text { as } \omega \rightarrow-\infty .
\end{aligned}
$$

Thus,

$$
G(\omega+m)=A_{1}(\omega) \omega^{-P-1}
$$

and

$$
H(\omega-n)=B_{1}(\omega) \omega^{-Q-1},
$$

where $A_{1}(\omega)$ and $B_{1}(\omega)$ are bounded as $\omega \rightarrow \pm \infty$. Let 


$$
r=P+Q+2 \text {. }
$$

Since by hypothesis, $-1<P+Q$, we know that $1<r$. From (4.11) and (4.12), we have

$$
|G(\omega+m)| \in L^{r /(P+1)}(-\infty, \infty) \text { and }|H(\omega-n)| \in L^{r /(\theta+1)}(-\infty, \infty) .
$$

Thus, we may apply Hölder's inequality [10, p. 382] and obtain

$$
\begin{aligned}
& \int_{-\infty}^{\infty}|G(\omega+m) H(\omega-n)| d \omega \\
& \quad \leqq\left\{\int_{-\infty}^{\infty}|G(\omega+m)|^{r /(P+1)} d \omega\right\}^{(P+1) / r}\left\{\int_{-\infty}^{\infty}|H(\omega-n)|^{r /(Q+1)} d \omega\right\}^{(Q+1) / r},
\end{aligned}
$$

since $(P+1) / r+(Q+1) / r=1$ by (4.13). But the R.H.S. of (4.14) is independent of $m$ and $n$ (since we can replace $\omega$ by $\omega-m$ in $G$ and by $\omega+n$ in $H$ ), and we denote it by the constant $M$. Thus, combining (4.10) and (4.14), we get

$$
\sum_{m, n=0}^{\infty}\left|A_{m n}\right| \int_{-\infty}^{\infty}|G H| d \omega \leqq M \sum_{m, n=0}^{\infty}\left|A_{m n}\right|
$$

and this last series converges. Thus, (4.8) is verified and the theorem is proved.

We complete our rigorous derivations by extending the Leibniz rule to the case in which we differentiate with respect to an arbitrary function $g(z)$. The proof of the following Corollary 4.1 follows from the integral analog of the Leibniz rule (4.1) in exactly the same way as the corresponding result [7, Corollary 4.2] follows from the series form of the Leibniz rule. Thus, we omit the proof.

COROllary 4.1. Assume the hypothesis of Theorem 4.1 and the additional conditions

(i) $g(z)$ is regular and univalent for $z \in g^{-1}(R)$,

(ii) $f_{*}(\xi, \zeta)=f(g(\xi), g(\zeta))$,

(iii) $\theta_{*}(\xi ; z)=\theta(g(\xi) ; g(z))=(g(\xi)-g(z)) q_{*}(\xi)$,

(iv) $q(g(\xi))=q_{*}(\xi)$.

Then,

$$
D_{o(z)}^{\alpha} f_{*}(z, z)=\int_{-\infty}^{\infty}\left(\begin{array}{c}
\alpha \\
\omega+\gamma
\end{array}\right)
$$

$$
\left.\cdot D_{o(\xi), \sigma(\zeta)}^{\alpha-\omega-\gamma, \omega+\gamma}\left[f_{*}(\xi, \zeta) \frac{d \theta_{*}(\zeta ; z)}{d \zeta} q_{*}(\xi)^{\omega+\gamma} q_{*}(\zeta)^{-\omega-\gamma-1}\right]\right|_{\xi=z ; \zeta=z} d \omega
$$

for $z \in g^{-1}(S)$, and all $\alpha$ and $\gamma$ for which $\left(\begin{array}{c}\alpha \\ \omega+\gamma\end{array}\right)$ is defined.

If, in addition, we have

(v) $f_{*}\left(\xi, g^{-1}(0)\right)=0$,

then (4.15) can be simplified to

$$
\begin{aligned}
D_{y(z)}^{\alpha} f_{*}(z, z)= & \int_{-\infty}^{\infty}\left(\begin{array}{c}
\alpha \\
\omega+\gamma
\end{array}\right) \\
& \left.\cdot D_{o(\xi), \rho(\zeta)}^{\alpha-\omega-\gamma, \omega+\gamma-1}\left[\frac{\partial f_{*}(\xi, \zeta)}{\partial g(\zeta)}\left[q_{*}(\xi) / q_{*}(\zeta)\right]^{\omega+\gamma}\right]\right|_{\xi=z ; \zeta=z} d \omega .
\end{aligned}
$$


Having completed our rigorous analysis, we now turn to the evaluation of definite integrals which are obtained from (4.15).

5. Examples. In this section, we give several examples of definite integrals obtained from (1.4) by selecting specific functions for $f(\xi, \zeta), q(\zeta), g(z)$ and specifying the parameters $\alpha$ and $\gamma$. Table 5.1 lists our choices for $f, q, g, \alpha, \gamma$ and Table 5.2 shows the resulting definite integrals. In computing the fractional derivatives in (1.4), use was made of the extensive table of Riemann-Liouville integrals found in [1, Vol. 2, pp. 181-200]. The notation used for the special functions is that of Erdélyi et al. [1].

Attention is called to the various restrictions appearing in Table 5.2. These are sometimes too strong. For example, it is well known that integral 8 requires only $3<\operatorname{Re}(A+B+C+D)$. The other two restrictions are not needed for the validity of the integral. They emerge from item (i) of the hypothesis of Theorem 4.1 in which we require $-1<\operatorname{Re}(P)$ and $-1<\operatorname{Re}(Q)$ so that $D_{\xi, \zeta}^{a, b} f(\xi, \zeta)$ is defined. Since Table 5.2 is provided to illustrate our integral form of the Leibniz rule, all restrictions emerging from the theorems of this paper are listed.

From the series form of the Leibniz rule (1.5), we see that in each integral in Table 5.2 we can replace " $\omega$ " by " $a n$ " and " $\int_{-\infty}^{\infty} \cdots d \omega$ " by " $\sum_{n=-\infty}^{\infty} \cdots a$ " to obtain a valid expression. Series forms of integrals 1 through 13 were given in [7].

TABLE 5.1 Choices for functions and parameters in the integral analog of the Leibniz rule (1.4) from which the definite integrals in Table 5.2 are derived.

\begin{tabular}{llllll} 
No. & $f(\xi, \zeta)$ & $q(\zeta)$ & $g(z)$ & $\alpha$ & $\gamma$ \\
\hline \hline 1 & $\zeta^{B-1}(1-\zeta)^{-A}$ & 1 & $z$ & $B-C$ & $\gamma$ \\
2 & $e^{\zeta} \zeta^{A-1}$ & 1 & $z$ & $A-B$ & $A-C$ \\
3 & $(\cos \zeta) / \zeta$ & 1 & $z^{2}$ & $-\nu-\frac{1}{2}$ & $-\frac{1}{2}-B$ \\
4 & $(\cosh \zeta) / \zeta$ & 1 & $z^{2}$ & $-\nu-\frac{1}{2}$ & $-\frac{1}{2}-B$ \\
5 & $(\sin \zeta) / \zeta$ & 1 & $z^{2}$ & $-\nu-\frac{1}{2}$ & $-\frac{1}{2}-B$ \\
6 & $(\sinh \zeta) / \zeta$ & 1 & $z^{2}$ & $-\nu-\frac{1}{2}$ & $-\frac{1}{2}-B$ \\
7 & $\left(1-\zeta^{2}\right)^{\nu}$ & 1 & $1-z$ & $\nu+\mu$ & $\gamma$ \\
8 & $\xi^{B+C-2} \zeta^{A+D-2}$ & 1 & $z$ & $A+C-2$ & $A-1$ \\
9 & $\xi^{b-1}(1-\xi)^{-e} \zeta^{B-1}(1-\zeta)^{-E}$ & 1 & $z$ & $b+B-d-D$ & $B-D$ \\
10 & $\xi^{D-A-1} \zeta^{C-B-1}(\xi \zeta+1)^{-E}$ & 1 & $z$ & $C-A-1$ & $C-1$ \\
11 & $\xi^{A-1} \zeta^{B}$ & $\zeta^{k}+P^{k}$ & $z$ & $\alpha$ & $\gamma$ \\
12 & $\xi^{A-1} \zeta^{B}$ & $\exp \left(\zeta^{k}\right)$ & $z$ & $\alpha$ & $\gamma$ \\
13 & $\xi^{A} \zeta^{B-1}{ }_{r} F_{s}\left(a_{1}, \cdots, a_{r} ;\right.$ & 1 & $z$ & $\alpha$ & $\gamma$ \\
& $\left.b_{1}, \cdots, b_{s} ; \zeta\right)$ & & & & $a-1$ \\
14 & $e^{\xi+\zeta} \xi^{b+c-2} \zeta^{a+d-2}$ & 1 & $z$ & $a+b-2$ & $\gamma$ \\
15 & $\xi^{p}\left(\xi^{k}+a^{k}\right)^{A} \zeta^{a}\left(\zeta^{k}+a^{k}\right)^{B}$ & 1 & $z$ & $\alpha$ & \\
\end{tabular}


TABLE 5.2 Definite integrals obtained from the integral analog of the Leibniz rule:

No.

Definite integral

$1 \frac{\pi_{2} F_{1}(A, B ; C ; z)}{\Gamma(C) \Gamma(B-C+1)}$

$$
=\int_{-\infty}^{\infty} \frac{\sin ((\omega+\gamma+C-B) \pi)_{2} F_{1}(A, B ; B-\gamma-\omega ; z)}{(\omega+\gamma+C-B) \Gamma(\omega+\gamma+1) \Gamma(B-\gamma-\omega)} d \omega,
$$

$\operatorname{Re}(z)<\frac{1}{2}, \quad 0<\operatorname{Re}(B)$.

$2 \frac{\pi_{1} F_{1}(A ; B ; z)}{\Gamma(B) \Gamma(A-B+1)}=\int_{-\infty}^{\infty} \frac{\sin ((\omega+B-C) \pi)_{1} F_{1}(A ; C-\omega ; z)}{(\omega+B-C) \Gamma(\omega+A-C+1) \Gamma(C-\omega)} d \omega$, $\operatorname{Re}(A)>0$.

3

$$
F_{\nu}(z)=\frac{\Gamma\left(\frac{1}{2}-\nu\right)}{\pi}\left(\frac{z}{2}\right)^{\nu-B} \int_{-\infty}^{\infty} \frac{\sin ((\omega+\nu-B) \pi) \mathscr{F}_{B-\omega}(z)}{(\omega+\nu-B) \Gamma\left(\omega-B+\frac{1}{2}\right)}\left(\frac{z}{2}\right)^{\omega} d \omega,
$$

through

6

where $\mathcal{F}_{v}=J_{v}, I_{v}, \mathrm{H}_{\nu}$ and $\mathrm{L}_{v}$, respectively, for series 3, 4, 5 and 6 .

$7 \quad \frac{\pi P_{\nu}^{\mu}(z)}{\Gamma(\nu+\mu+1)}=\int_{-\infty}^{\infty} \frac{\sin ((\omega+\gamma-\nu-\mu) \pi)}{(\omega+\gamma-\nu-\mu)}$

$$
\frac{P_{\nu}^{\omega+\gamma-\nu}(z)}{\Gamma(\omega+\gamma+1)}\left(\frac{1-z}{1+z}\right)^{(\omega+\gamma-\nu-\mu) / 2} d \omega
$$

$-1<\operatorname{Re}(\nu), \quad 0<\operatorname{Re}(z)$.

$8 \frac{\Gamma(A+B+C+D-3)}{\Gamma(A+C-1) \Gamma(A+D-1) \Gamma(B+C-1) \Gamma(B+D-1)}$

$$
=\int_{-\infty}^{\infty} \frac{1}{\Gamma(\omega+A) \Gamma(\omega+B) \Gamma(C-\omega) \Gamma(D-\omega)} d \omega,
$$

$1<\operatorname{Re}(B+C), \quad 1<\operatorname{Re}(A+D), \quad 3<\operatorname{Re}(A+B+C+D)$.

$9 \quad \frac{\Gamma(b+B-1){ }_{2} F_{1}(e+E, b+B-1 ; d+D-1 ; z)}{\Gamma(d+D-1) \Gamma(b+B-d-D+1) \Gamma(b) \Gamma(B)}$

$$
\begin{gathered}
=\int_{-\infty}^{\infty} \frac{{ }_{2} F_{1}(e, b ; d+\omega ; z){ }_{2} F_{1}(E, B ; D-\omega ; z)}{\Gamma(\omega+B-D+1) \Gamma(\omega+d) \Gamma(b-d-\omega+1) \Gamma(D-\omega)} d \omega, \\
0<\operatorname{Re}(b), \quad 0<\operatorname{Re}(B), \quad \frac{1}{2}>\operatorname{Re}(z), \quad 1<\operatorname{Re}(b+B) .
\end{gathered}
$$




\section{TABLE 5.2 (continued)}

No.

Definite integral

$$
\begin{aligned}
& \frac{\Gamma(C+D-A-B-1)_{3} F_{2}\left[\begin{array}{c}
E,(C+D-A-B-1) / 2,(C+D-A-B) / 2 ;-z^{2} \\
(D-B) / 2,(D-B+1) / 2
\end{array}\right]}{\Gamma(C-A) \Gamma(C-B) \Gamma(D-A) \Gamma(D-B)}
\end{aligned}
$$

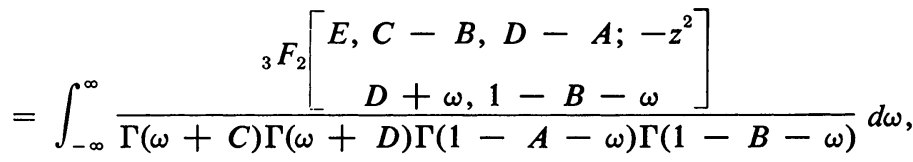

$$
\begin{aligned}
& 0<\operatorname{Re}(D-A), \quad 0<\operatorname{Re}(C-B), \quad 1<\operatorname{Re}(C+D-A-B) \text {. }
\end{aligned}
$$

11

$$
\begin{aligned}
& \frac{\Gamma(A+B)}{\Gamma(A+B-\alpha) \Gamma(\alpha+1) \Gamma(A) \Gamma(B+1)} \\
& \quad \int_{-\infty}^{\infty} \frac{{ }^{k+1} F_{k}\left[\begin{array}{c}
-\omega-\gamma,\{A\}_{k} ;-z^{k} / P^{k} \\
(\omega+A+\gamma-\alpha\}_{k}
\end{array}\right]{ }_{k+1} F_{k}\left[\begin{array}{c}
\omega+\gamma,\{B\}_{k} ;-z^{k} / P^{k} \\
\{B-\omega-\gamma+1\}_{k}
\end{array}\right] d \omega}{\Gamma(\omega+\gamma+1) \Gamma(\omega+A+\gamma-\alpha) \Gamma(\alpha-\gamma-\omega+1) \Gamma(B-\gamma-\omega+1)}, * \\
& -1<\operatorname{Re}(A), \quad 0<\operatorname{Re}(B), \quad k=1,2,3, \cdots .
\end{aligned}
$$

12

$$
\Gamma(A+B)
$$

$\overline{\Gamma(A+B-\alpha) \Gamma(\alpha+1) \Gamma(A) \Gamma(B+1)}$

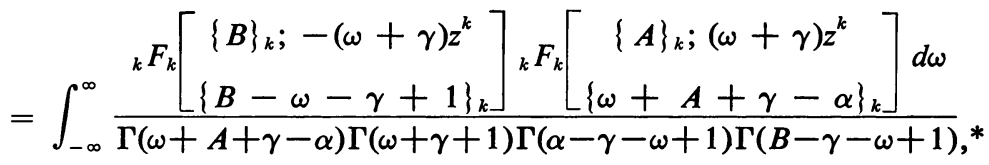

$-1<\operatorname{Re}(A), \quad 0<\operatorname{Re}(B), \quad k=1,2,3, \cdots$.

13

$\frac{\Gamma(A+B)_{r+1} F_{s+1}\left[\begin{array}{c}A+B, a_{1}, \cdots, a_{r} ; z \\ A+B-\alpha, b_{1}, \cdots, b_{s}\end{array}\right]}{\Gamma(A+B-\alpha) \Gamma(\alpha+1) \Gamma(A+1) \Gamma(B)}$

$$
=\int_{-\infty}^{\infty} \frac{{ }_{r+1} F_{s+1}\left[\begin{array}{c}
B, a_{1}, \cdots, a_{r} ; z \\
B-\gamma-\omega, b_{1}, \cdots, b_{s}
\end{array}\right]}{\Gamma(\omega+\gamma+1) \Gamma(\omega+A-\alpha+\gamma+1) \Gamma(\alpha-\gamma-\omega+1) \Gamma(B-\gamma-\omega)} d \omega,
$$

$-1<\operatorname{Re}(A), \quad 0<\operatorname{Re}(B)$.

* The notation $\{A\}_{k}$ stands for the set of $k$ parameters $A / k,(A+1) / k,(A+2) / k$, $\cdots,(A+k-1) / k$. 
TABLE 5.2 (continued)

No.

Definite integral

14

$$
\begin{aligned}
& \frac{\Gamma(a+b+c+d-3)_{1} F_{1}(a+b+c+d-3 ; c+d-1 ; 2 z)}{\Gamma(c+d-1) \Gamma(b+c-1) \Gamma(a+b-1) \Gamma(a+d-1)} \\
& =\int_{-\infty}^{\infty} \frac{{ }_{1} F_{1}(b+c-1 ; c+\omega ; z){ }_{1} F_{1}(a+d-1 ; d-\omega ; z) d \omega}{\Gamma(a+\omega) \Gamma(b-\omega) \Gamma(c+\omega) \Gamma(d-\omega)},
\end{aligned}
$$

$3<\operatorname{Re}(a+b+c+d), \quad 1<\operatorname{Re}(b+c), \quad 1<\operatorname{Re}(a+d)$.

15

$$
\begin{aligned}
& \frac{\Gamma(p+q+1)_{k+1} F_{k}\left[\begin{array}{c}
\left.-A-B,\{p+q+1\}_{k} ;-z^{k} / a^{k}\right] \\
\{p+q-\alpha+1\}_{k}
\end{array}\right]}{\Gamma(p+q-\alpha+1) \Gamma(\alpha+1) \Gamma(p+1) \Gamma(q+1)} \\
& =\int_{-\infty}^{\infty} \frac{{ }^{k+1} F_{k}\left[\begin{array}{c}
-A,\{p+1\}_{k} ;-z^{k} / a^{k} \\
\{p-\alpha+\gamma+\omega+1\}_{k}
\end{array}\right]_{k+1} F_{k}\left[\begin{array}{c}
\left.-B,\{q+1\}_{k} ;-z^{k} / a^{k}\right] \\
\Gamma(\alpha-\gamma-\omega+1) \Gamma(\omega+\gamma+1) \Gamma(p-\alpha+\gamma+\omega+1) \Gamma(q-\gamma-\omega+1)
\end{array}\right.}{\Gamma-\gamma}, \\
& -1<\operatorname{Re}(p), \quad-1<\operatorname{Re}(q), \quad-1<\operatorname{Re}(p+q), \quad k=1,2,3, \cdots .
\end{aligned}
$$

Department of Mathematics

Rensselaer Polytechnic Institute

Troy, New York 12181

1. A. Erdélyi, W. Magnus, F. Oberhettinger \& F. G. Tricomi, Tables of Integral Transforms. Vols. 1, 2, McGraw-Hill, New York, 1954, 1955. MR 15, 868; MR 16, 586.

2. Y. L. LUKe, The Special Functions and Their Approximations. Vols. 1, 2, Math. in Sci. and Engineering, vol. 53, Academic Press, New York, 1969. MR 39 \#3039; MR 40 \#2909.

3. T. J. OsLer, "Leibniz rule for fractional derivatives generalized and an application to infinite series," SIAM J. Appl. Math., v. 18, 1970, pp. 658-674. MR 41 \#5562.

4. T. J. OsLer, "The fractional derivative of a composite function," SIAM J. Math. Anal., v. 1, 1970, pp. 288-293. MR 41 \#5563.

5. T. J. OSLER, "Taylor's series generalized for fractional derivatives and applications," SIAM J. Math. Anal., v. 2, 1971, pp. 37-48.

6. T. J. Osler, "Fractional derivatives and Leibniz rule," Amer. Math. Monthly, v. 78, 1971, pp. 645-649.

7. T. J. OsLer, "A further extension of the Leibniz rule to fractional derivatives and its relation to Parseval's formula," SIAM J. Math. Anal., v. 3, 1972, pp. 1-16.

8. T. J. OsLer, "An integral analogue of Taylor's series and its use in computing Fourier transforms," Math. Comp., v. 26, 1972, pp. 449-460.

9. T. J. OsLER, "A correction to Leibniz rule for fractional derivatives." (To appear.) 1939.

10. E. C. Titchmarsh, The Theory of Functions, 2nd ed., Oxford Univ. Press, London,

11. E. C. TITchmaRsh, Introduction to the Theory of Fourier Integrals, 2nd ed., Clarendon Press, Oxford, 1948. 